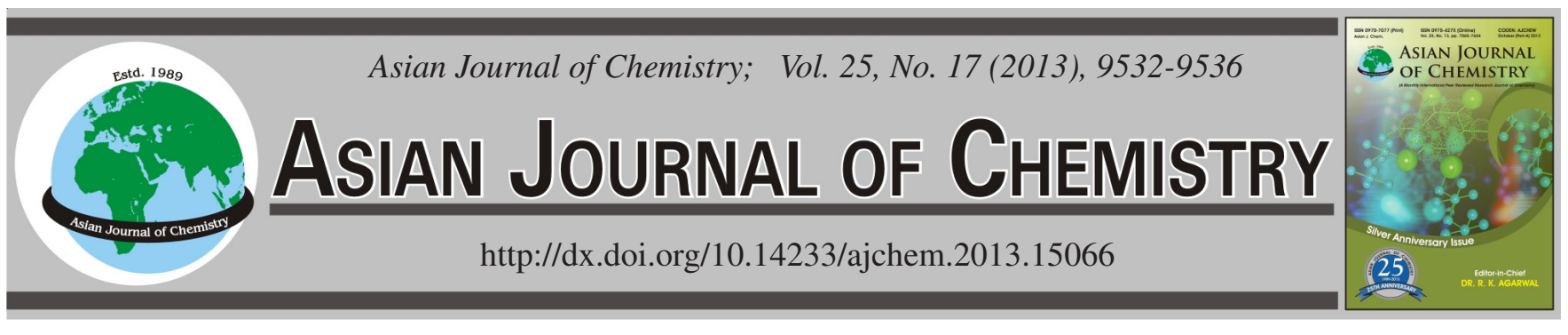

\title{
Preparation and Characterization of Folate Modified- Chitosan Nanoparticles as New Tumor-Targeting Drug Carrier
}

\author{
ZHI-HuA XING
}

College of Pharmacy, Harbin University of Commerce, Harbin 150076, P.R. China

Corresponding author: E-mail: yikaiwang@foxmail.com; xzhh0451@163.com

\begin{abstract}
In order to enhance the tumor-targeting and anticancer activity of drugs and prolong the total duration of action, doxorubicin (DOX) loaded foliate (FA)-decorated chitosan (CTS) nanoparticles (FA-CTS/DOX) were prepared by the ionic crosslinking method through the electrostatic interaction between the protonated amino group of chitosan in acidic conditions and the deprotonation of carboxyl group of folic acid in alkaline conditions as well as sodium tripolyphosphate (TPP). The morphological characteristics of nanoparticles were examined using transmitting electron microscope. The average particle size of nanoparticles and size distribution were determined by dynamic light scattering. The drug encapsulation efficiency, loading capacity and in vitro release characteristics were investigated using ultraviolet spectrophotometer. The results indicated that the nanoparticles are uniform spherical and regular, with a size of $145 \mathrm{~nm},+40$ $\mathrm{mV}$ surface potential and good size stability. Its encapsulation efficiency and loading capacity were up to 85.9 and $14.9 \%$, respectively. The Higuchi equation: $\mathrm{Q}=14.546 \mathrm{t}^{1 / 2}+8.2579\left(\mathrm{R}^{2}=0.9687\right)$ (in PBS buffer solution of $\left.\mathrm{pH}=7.4\right)$ can well describe release curve of the FA-CTS/DOX nanoparticles. It was inferred that the CTS-FA/DOX nanoparticles should posses good tumor targeting from the average particle size of $145 \mathrm{~nm}$ and the amount of folic acid conjugated with chitosan (CTS:FA $=1: 17$ ).
\end{abstract}

Key Words: Chitosan, Folate, Nanoparticles, Ionic crosslinking, Tumor-targeting.

\section{INTRODUCTION}

Chitosan (CTS) is currently the only known alkaline polysaccharide with positive charges. It has good biocompatibility, biodegradability, non-toxic and biological adhesion, antibacterial antiinflammatory, wound healing, acid-resistant and direct inhibitory effect of tumor cells, combined with existing anticancer drugs to enhance anticancer effects. In addition, chitosan has extensive sources and low price. It has been considered as an ideal drug delivery material ${ }^{1,2}$.

Recent studies have shown that the foliate receptor (FR), a glycosyl-phosphatidylinositol (GPI) anchored cell surface, has been known to be overexpressed in about $30 \%$ of human malignant tumors, including breast and ovarian cancers, but hardly seen in normal tissues ${ }^{3}$. It is well known that folic acid (FA), a vitamin has high-level affinity to the foliate receptor. Taking folic acid as targeting ligand was a strategy by which anticancer drugs were targeted to the tumor, thus increasing the therapeutic index of the drug, was a way of improving cancer therapy and minimizing systematic toxicity ${ }^{4-7}$.

Doxorubicin is an effective chemotherapeutic agent in different cancers and widely used in clinical field. In spite of its success against many cancers, the short biological half life, nonspecific distribution leading to intolerable adverse effects and development of drug resistance have limited its usefulness. Few polymers based delivery systems developed for dioxorubicin are mostly designed to reduce or alter toxicity ${ }^{8-10}$.

In recent years, the domestic and foreign scholars prepared folic acid-chitosan micro/nano-particles mostly adopting coupling method ${ }^{4-7}$. That was, active ester of folic acid reacted with amino group of chitosan to obtain coupling folic acidchitosan, then, reacted with sodium trimeric phosphate (TPP) to obtain folic acid-chitosan micro/nano-particles through ionic crosslinking and finally embeded the anti- cancer medicine through the adsorption law. During the coupling reaction of folic acid and chitosan, the massive organic reagents and toxic coupling reagent such as DCC must be used. The residual coupling reagent will inevitably resulted in the cell toxicity to organisms as well as the deactivation to the macro-molecule. There were many shortcomings of the encapsulation efficiency and low sample volume in the method of adsorption of drug entrapped.

In this study, dioxorubicin is selected as a drug model, chitosan as drug vector, folic acid as tumor targeting ligand and sodium trimeric phosphate as polyanion to directly prepare folic acid-chitosan nanoparticles in water by ionic 
crosslinking method through electrostatic interactions between protonated amino group of chitosan molecular chain in acidic environment and deprotonation carboxyl group of foliate molecular structure in alkalinity environment as well as FACTS/DOX nanoparticles no using any organic reagent and toxic coupling reagent. In a word, this method is of green synthesis technology, no pollution, no toxic organic reagents, environment friendly, simple steps and low cost.

\section{EXPERIMENTAL}

Folic acid (sigma); Chitosan (Jinan Hyderabad Beihai Ocean Bio-Engineering Co., Ltd., degree of deacetylation > $95 \%$, relative molecular mass 50000); Doxorubicin (Shanghai Bio-Technology Co., Ltd.). All other reagents were of analytical grade.

\section{Preparation of FA-CTS and FA-CTS/DOX nanoparticles}

Preparing solutions of chitosan, folic acid and sodium trimeric phosphate: Solutions of chitosan $\left(2.0 \mathrm{mg} \mathrm{mL}^{-1}\right)$ was prepared in $36 \%$ acetic acid whose concentration was $7.5 \mathrm{mg}$ $\mathrm{mL}^{-1}$ at room temperature and with intensive stirring for $0.5 \mathrm{~h}$.

Solution of folic acid $\left(1.0 \mathrm{mg} \mathrm{mL}^{-1}\right)$ was prepared in $25 \%$ ammonia whose concentration was $2.5 \mathrm{mg} \mathrm{mL}^{-1}$ at room temperature and with intensive stirring for $10 \mathrm{~min}$.

To form nanoparticles by ionic crosslinking, sodium trimeric phosphate $\left(2.0 \mathrm{mg} \mathrm{mL}^{-1}\right)$ prepaed in deionized water was used. The resulting solutions were put through a filter with a pore size of $3 \mathrm{~m}$.

Formation of nanoparticles: FA-CTS/DOX nanoparticles were prepared by ionic crosslinking and described as follows: $50 \mathrm{~mL}$ of folic acid solution $\left(1.0 \mathrm{mg} \mathrm{mL}^{-1}\right)$ and $20 \mathrm{~mL}$ of sodium trimeric phosphate solution $\left(2.0 \mathrm{mg} \mathrm{mL}^{-1}\right)$ were added dropwise to $200 \mathrm{~mL}$ of chitosan solution $\left(2 \mathrm{mg} \mathrm{mL}^{-1}\right)$ containing $90 \mathrm{mg}$ dioxorubicin in sequence, at $55^{\circ} \mathrm{C}$ with magnetic stirring (900-1000 rpm). The dispersion that spontaneously formed was stirred for $0.5 \mathrm{~h}$. The particles were separated by centrifugation (3000 rpm, $20 \mathrm{~min}$ ) from the dispersion, resuspended in deionized water and newly centrifuged. Then, the yellow precipitate was collected, lyophilized and ready for the further investigation. To avoid the nanoparticles gathering, appropriate amount of mannose was added to the yellow precipitate when they were lyophilized.

For the need of blank control, folic acid-chitosan nanoparticles were prepared identically to the above method, but without dioxorubicin.

FTIR measurement: The structure characterization of folic acid-chitosan particles was determined using FTIR-650 Fourier transform infrared spectroscopy (Tianjin Port East Technology). The sample was made into a tablet with $\mathrm{KBr}$ at room temperature and the same preparation of chitosan and folate sample were made as well for comparison.

Average particle size and surface morphology of nanoparticles: The particle size and size distribution were determined by the method of inverse light dispersion using LS13320 laser particle size analyzer (US Beckman Coulter Co.).

The surface morphology of particles was determined by transmission electron microscopy method. The specific method was as follows: The water dispersion system of FA-CTS/DOX nanoparticles was uniformly dropped to the copper net of TEM for sample preparation and negatively stained with $2 \%$ phosphotungstic acid. The surfaces of the particles were observed and the pictures were taken via using Hitachi-7650 TEM (Japan) after moisture evaporated and dried.

Surface potential of nanoparticles: Take proper amount of nanoparticles suspension, add deionized water to dilute it, then determine the surface potential of particles using Zetasizer Nano Z potential analyzer (Malvern of Britain Co.).

\section{Performance evaluation of nanoparticles}

Dynamics of the stability of nanoparticles: Samples of nanoparticles were kept for 45 days in conventional conditions at $23 \pm 2{ }^{\circ} \mathrm{C}$. During the time, the average particle size were determined in 7, 15, 30 and 45 days, individually.

Targeting of nanoparticles: The passive targeting of FACTS/DOX nanoparticles is depended on the size of particles and the active targeting is depended on how much folate was conjugated by ionic bond with chitosan, To determine the content of FA in the particles, the FA-CTS/DOX nanoparticles water dispersion was centrifuged at $3000 \mathrm{rpm}$ for $0.5 \mathrm{~h}$ and the amount of free folate in the supernatant was measured by the standard curve method using a Tu-1800PC UV-visible spectrophotometer (Beijing Purkinje General Instrument Co., Ltd.) at $365 \mathrm{~nm}$. The amount of substance ratio of CTS to FA was calculated according to the following formula:

$$
\frac{\text { Chitosan }}{\text { Folic acid }}=\frac{\text { Total chitosan }(\mathrm{mol})}{\text { Total folic acid }(\mathrm{mol})-\text { Free folic acid }(\mathrm{mol})}
$$

Sorption of dioxorubicin by nanoparticles: To determine the entrapment efficiency and the loading capacity, the FACTS/DOX nanoparticles were centrifuged at $3000 \mathrm{rpm}$ for 0.5 $\mathrm{h}$ from the dispersion, resuspended in the $\mathrm{pH} 5.0$ acetic acid solution and newly centrifuged. The amount of free dioxorubicin in the supernatant was measured by the standard curve method using a UV-visible spectrophotometer at 284 $\mathrm{nm}$. The precipitate was then lyophilized and weighed. The dioxorubicin entrapment efficiency (EE) and the loading capacity (LC) were calculated according to the following formula:

$$
\begin{gathered}
\mathrm{EE}=\frac{\text { Total DOX }(\mathrm{mg})-\text { Free DOX }(\mathrm{mg})}{\text { Total DOX }(\mathrm{mg})} \times 100 \% \\
\mathrm{LC}=\frac{\text { Total DOX }(\mathrm{mg})-\text { Free DOX }(\mathrm{mg})}{\text { Nanoparticles Weight }(\mathrm{mg})} \times 100 \%
\end{gathered}
$$

All measurements were performed in triplicate and averaged to obtain reliable results.

In vitro release studies: The release behaviour of nanoparticles was studied by cylindrical basket method. The release medium are $\mathrm{pH} 1.2$ artificial gastric juices, $\mathrm{pH} 6.8$ intestinal juice and $\mathrm{pH} 7.4$ artificial body fluid and temperature is $37 \pm 1^{\circ} \mathrm{C}$. At predetermined time-points, the receiving fluid was withdrawn and the release study was continued after replacement with the same amont of fresh buffer. The amount of dioxorubicin released was determined by the standard curve method using a UV-visible spectrophotometer at $284 \mathrm{~nm}$ and a calibration curve was made using none-loaded nanoparticles to correct for the intrinsic absorption of the polymer. In each experiment, the samples were analyzed in triplicate and the error bars represent the standard deviation. 


\section{RESULTS AND DISCUSSION}

IR spectra of nanoparticles: IR spectra was shown in Fig. 1. Fig. 1 indicated that folic acid-chitosan complexes were in conjunction through electrostatic interactions between protonated amino group of chitosan molecular chain and deprotonation carboxyl of folic acid molecular structure. On one hand, the aromatic ring skeleton to expand and contract vibration peak 1604 and $1485 \mathrm{~cm}^{-1}$ appeared in the polymer, it indicated that folic acid and chitosan combined with success; on the other hand, $1705 \mathrm{~cm}^{-1}$ carbonyl peaks of folic acid $(-\mathrm{COOH})$ disappeared, that indicated the carboxyl of folic acid molecule and amino group of chitosan were combined with ionic bond, namely folic acid-chitosan complexes were in conjunction through electrostatic interactions.

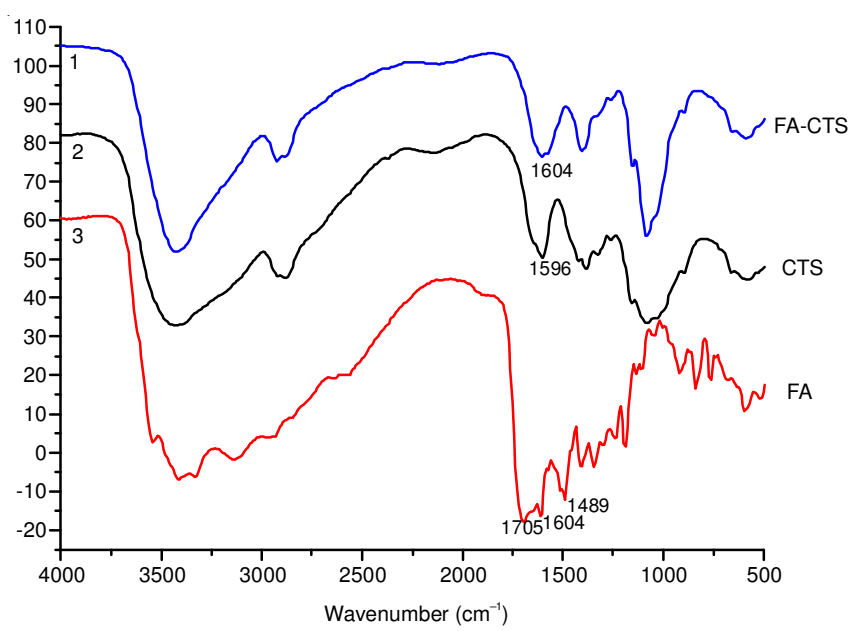

Fig. 1. IR spectra of folic acid-chitosan, chitosan, folic acid

Average particle size and surface morphology of nanoparticles: Measured by laser particle size analyzer, the data of FA-CTS/DOX nanoparticles was as follows: D[4,3] = $145 \mathrm{~nm}, \mathrm{~d}(0.1)=110 \mathrm{~nm}, \mathrm{~d}(0.5)=142, \mathrm{~d}(0.9)=184 \mathrm{~nm}$, particle size dispersion index $(\mathrm{PI})=1.018$. The particle size distribution of FA-CTS/DOX nanoparticles was shown in Fig. 2.

The morphology characterization of nanoparticles was determined by the TEM. The photo of FA-CTS/DOX nanoparticles was shown in Fig. 3. It can be seen from the photo, the nanoparticles showed a regular spher with uniform size and no adhesion.

Surface potential of nanoparticles: Amino group $\left(-\mathrm{NH}_{2}\right)$ of chitosan transform into ammonia ion $\left(-\mathrm{NH}_{3}{ }^{+}\right)$under acidic conditions. Although chitosan react with FA as well as sodium trimeric phosphate and finally forming folic acid-chitosan nanoparticles, plenty of $-\mathrm{NH}_{3}{ }^{+}$were still remained in chitosan because of excess chitosan. so the surface of nanoparticles should posses positive charge and the surface potential was high. The experimental result shown that the surface potential of FA-CTS/DOX nanoparticles was $+40 \mathrm{mV}$ which accod with the above deduction. Generally, if the absolute value of surface potential of nanoparticles is more than $25 \mathrm{mV}$, nanoparticles will not gather in suspension due to the repellent action among the electrostatic force. At the same time, the surface of FA-CTS/DOX nanoparticles has positive charge which is

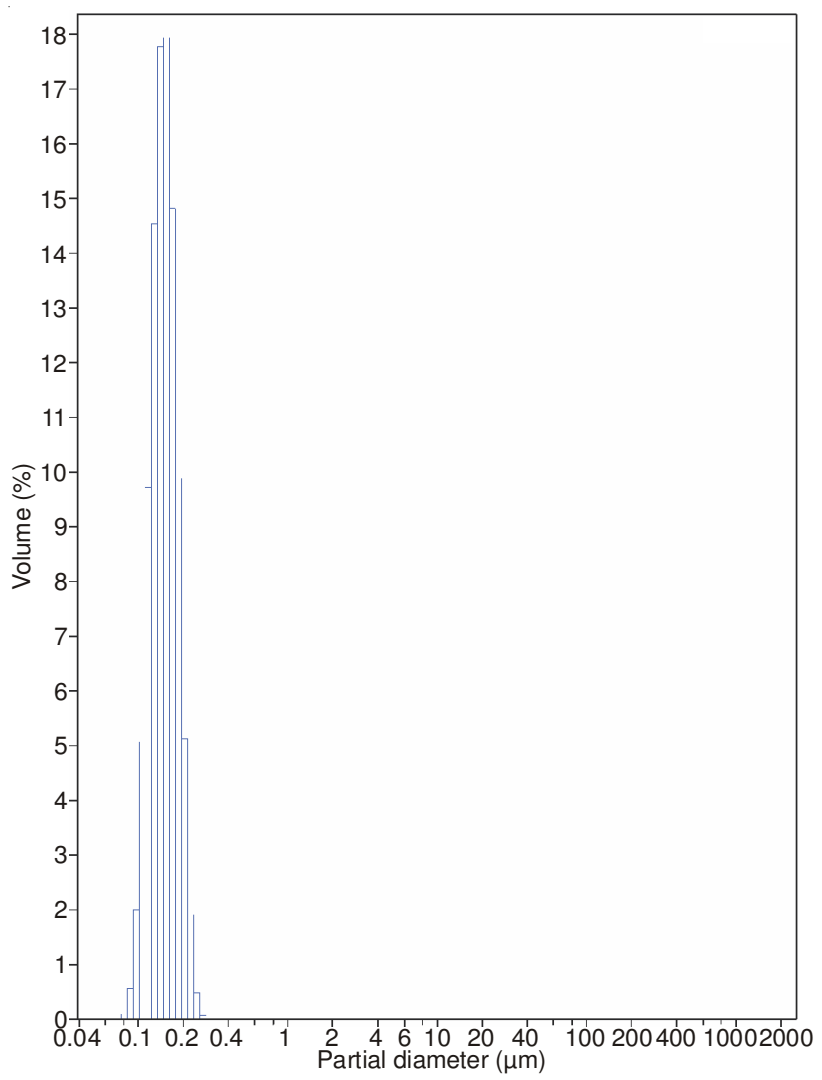

Fig. 2. Particle size distribution of FA-CTS/DOX nanoparticles

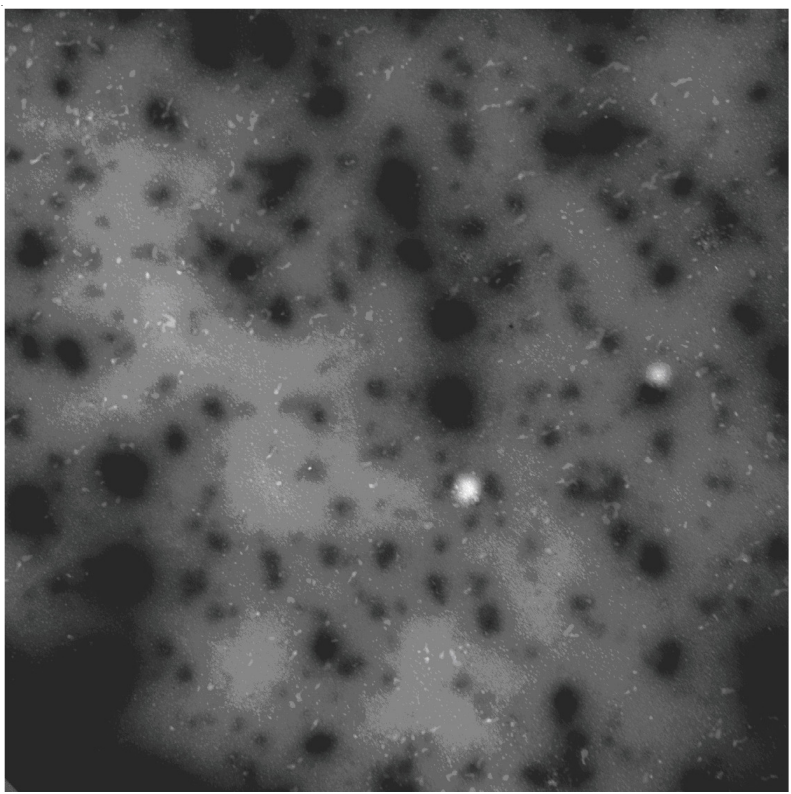

Fig. 3. TEM of FA-CTS/DOX nanoparticles

easily attracted by the negative charge of cellular membrane. It is beneficial for dioxorubicin to distribute in vivo through nanoparticle delivery and improve the treatment of the solid tumor.

Size stability of nanoparticles: The value of average particle size in different time is shown in Table-1. It can be seen from Table-1, the average particle size almost not change in 7 to 45 days, which indicated that the size stability of the FA-CTS/DOX nanoparticles was well owing to the high surface potential. 
TABLE-1

VALUE OF PARTICLE SIZE IN DIFFERENT TIME

\begin{tabular}{cc}
\hline Storage time $(\mathrm{d})$ & Particle size $(\mathrm{nm})$ \\
\hline 0 & 145 \\
7 & 145 \\
15 & 145 \\
30 & 146 \\
45 & 146 \\
\hline
\end{tabular}

Targeting of nanoparticles: Analysis of passive targeting: passive targeting is formed by natural distribution in vivo due to normal physiological function of organism. Generally, $100-200 \mathrm{~nm}$ of nanoparticles will not be intercepted by the sinusoidal vessel of liver and spleen ${ }^{11}$, so the FA-CTS/DOX nanoparticles with average particle size of $145 \mathrm{~nm}$ could enter blood circulation without interception by the sinusoidal vessel of liver and spleen and its retention time was prolonged. As a result, the chance that dioxorubicin embedded in the nanoparticles getting into the lesion location of tumor is increased.

Analysis of active targeting: The ratio of chitosan to folic acid is $1: 17$, namely one molecule of chitosan was conjugated with 17 molecules of folic acid. It shown that the folic acidchitosan nanoparticles have intensive tumor targeting according to the reference ${ }^{3,4,12}$ : If one molecule of protein was conjugated with 3 molecules of folate in the conjugation of folic acid and protein on average, it shown great tumor targeting. From this, the FA-CTS/DOX nanoparticles should posses strong active tumor targeting.

Encapsulation efficiency and loading capacity: FACTS/DOX nanopartcles were prepared repeated three groups to obtain reliable results. The average encapsulation efficiency was $(85.8 \pm 0.1) \%$ and the average loading capacity was $(14.6$ $\pm 0.3) \%$. It indicated that the folic acid-chitosan nanopartcles had not only high encapsulation efficiency to reduce the loss of dioxorubicin loaded but also good loading capacity to ensure the therapy and reduce the dosage of nanopartcles, moreover the nanopartcles preparation had good repeatability.

In order to obtain high encapsulation efficiency of drugloaded nanopartcles, we tried adsorption and mixing two methods. The adsorption method was: first, folic acid-chitosan nanopartcles water dispersion system was prepared, then appropriate amount of dioxorubicin was added to obtain loaded folic acid-chitosan nanopartcles. The mixing method was: a certain amount of dioxorubicin was suspended in the chitosan dilute acetic acid solution and then slowly added folic acid ammonium salt solution and sodium trimeric phosphate solution in sequence. The research result discovered that, the drug loading and the encapsulation efficiency of nanopartcles prepared by mixing method was much higher than that by the adsorption method. We therefore adopted mixing method for embedding of dioxorubicin.

Dioxorubicin in vitro release and discussion on the mechanism: Fig. 4 showed that FA-CTS/DOX nanoparticles had a good slow-release effect in artificial gastric juice. In artificial gastric juice, cumulative release of dioxorubicin was $7.50 \%$ within $15 \mathrm{~min}, 8.30 \%$ within $0.5 \mathrm{~h}, 17.01 \%$ within $1 \mathrm{~h}, 49.84 \%$ within $3 \mathrm{~h}$ and $75.43 \%$ within $4 \mathrm{~h}$. That closed to uniform speed release.

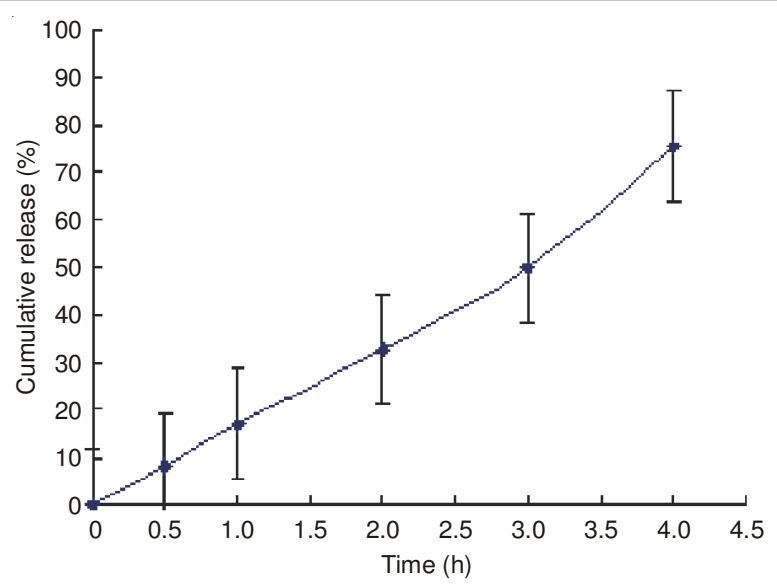

Fig. 4. Dioxorubicin release curve of load dioxorubicin nanoparticles in vitro (artificial gastric juice of $\mathrm{pH}=1.2$ )

In artificial intestinal juice, the cumulative release of dioxorubicin from FA-CTS/DOX nanoparticles is displayed in Fig. 5. The overall release process of dioxorubicin was a levelling-off release phase. The burst effect is minimal which was slight. Early release was not cumulative, that indicated the combination of drugs in the nanoparticles surface was relatively fewer, the vast majority of drugs were inside of nanoparticles. $5 \mathrm{~h}$ later dox released slowly due to swelling or degradation of chitosan and its maximum cumulative released quantity was up to $90.15 \%$ within $9 \mathrm{~h}$. Therefore, nanoparticles had good slow release function and almost no burst-releasing phenomena. Early nearly uniform release of dioxorubicin proved that the drug encapsulated in nanoparticles had played a certain controlled release effect.

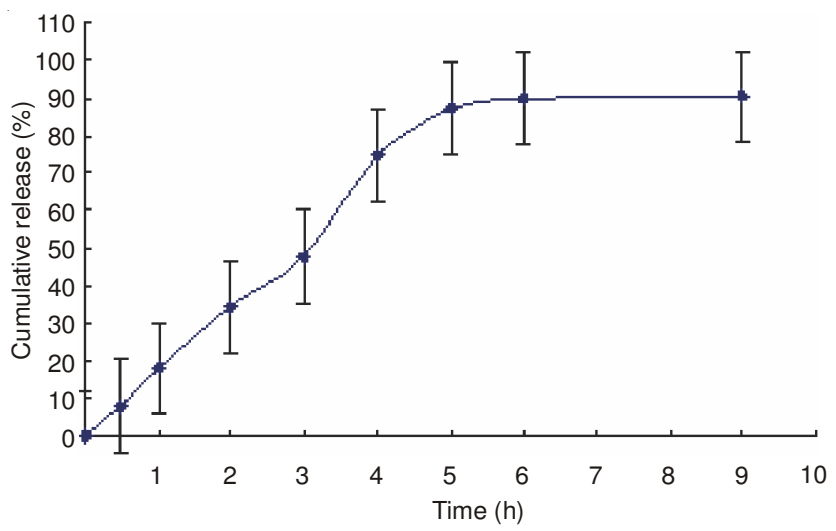

Fig. 5. Dioxorubicin release curve of load dioxorubicin nanoparticles in vitro (artificial intestinal juice of $\mathrm{pH}=6.8$ )

It can be seen from Fig. 6 that the nanoparticles had played good sustained release effect for dioxorubicin encapsulated in nanoparticles in PBS buffer solution of $\mathrm{pH}=7.4$. In initial stage, dioxorubicin released fast with the cumulative release amount ranged from $7.0 \%$ in $0.5 \mathrm{~h}$ to $32.8 \%$ in $3 \mathrm{~h}$. In the medium stage, dioxorubicin released nearly uniform from 3-24 h with $85 \%$ of cumulative release amount. In the late stage, dioxorubicin released very slowly and almost finished by $26 \mathrm{~h}$. About $15 \%$ of dioxorubicin were tightly embedded in the chitosan nanoparticles. Only did the chitosan nanoparticles occur corrosion or chitosan degrade, the $15 \%$ of dioxorubicin can release. However, known from the size 


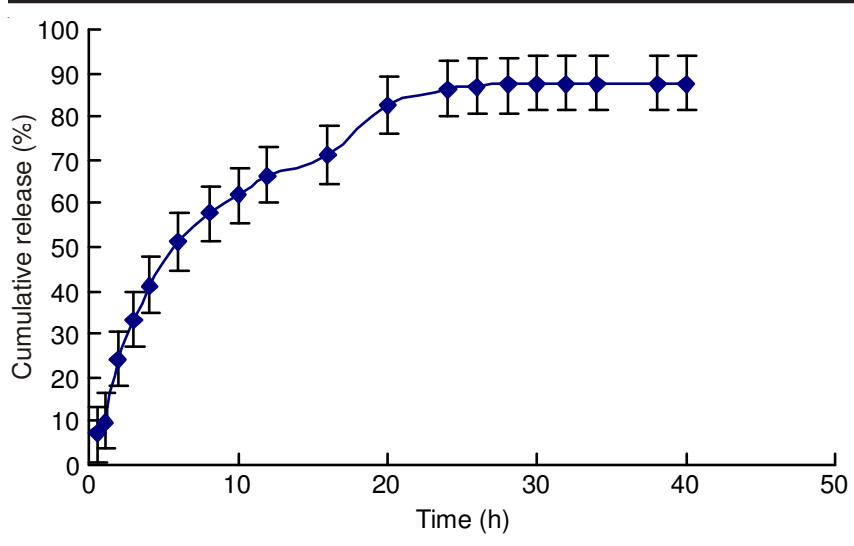

Fig. 6. Dioxorubicin release curve of load dioxorubicin nanoparticles in vitro (PBS buffer solution of $\mathrm{pH}=7.4$ )

stability of nanoparticles, not only did the chitosan nanoparticles occur corrosion too slow but also chitosan degrade too hard without corresponding enzyme. It can be inferred that dioxorubicin released maybe through leaching effect.

In order to evaluate FA-CTS/DOX nanoparticles in vitro release mechanism, curve of vitro release was fitted according to zero order kinetics, one order kinetics and Higuch equation, respectively. The results were shown in Table- 2 .

\begin{tabular}{ccc}
\multicolumn{3}{c}{ TABLE-2 } \\
\multicolumn{3}{c}{ FITTING EQUATION OF DATA ABOUT } \\
CUMULATIVE RELEASE IN PBS \\
\hline Name & Equation & $\mathrm{R}^{2}$ \\
\hline Zero order kinetics & $\mathrm{Q}=1.8345 \mathrm{t}+31.018$ & 0.7964 \\
One order kinetics & $\ln [1-\mathrm{Q}]=-0.0423 \mathrm{t}-5.1575$ & 0.5499 \\
Higuchi equation & $\mathrm{Q}=14.546 \mathrm{t}^{1 / 2}+8.2579$ & 0.9687 \\
\hline
\end{tabular}

The curve of dioxorubicin in vitro release in PBS buffer could be better described through Higuchi equations. That was, the relationship between release of dioxorubicin and the square root of time was a straight line, which conformed to drug release performance of the water-insoluble skeleton.

\section{Conclusion}

Dioxorubicin-loaded folate-decorated chitosan nanoparticles were prepared by ionic crosslinking method. The nanoparticles are uniform spherical and regular, with a size of $145 \mathrm{~nm},+40 \mathrm{mV}$ surface potential and good size stability. Its encapsulation efficiency and loading capacity were up to 85.9 and $14.9 \%$, respectively. The Higuchi equation: $\mathrm{Q}=14.546 \mathrm{t}^{1 / 2}$ $+8.2579\left(\mathrm{R}^{2}=0.9687\right)($ in PBS buffer solution of $\mathrm{pH}=7.4$ ) can well describe release curve of the FA-CTS/DOX nanoparticles. It was inferred that the CTS-FA/DOX nanoparticles should posses good tumor targeting from the average particle size of $145 \mathrm{~nm}$ and the amount of folic acid conjugated with chitosan $(\mathrm{CTS}: \mathrm{FA}=1: 17)$.

\section{ACKNOWLEDGEMENTS}

This work was financially supported by program for the Scientific Research Project of Education Department of Heilongjiang Province, China (12531156).

\section{REFERENCES}

1. C.P. Leamon and P.S. Low, Drug Discov. Today, 6, 44 (2001).

2. Zhang Li-Guo, Pan Ji-Lun, Li Jie-Liang et al., J. Funct. Mater, 35, 644 (2004).

3. X. Zhao, H. Li and R.J. Lee, Expert Opin Drug Deliv., 5, 309 (2008).

4. C.P. Leamon, Drug Delivery Systems, 6, 44 (2001).

5. Z. Ying, Y. Yan, L. Shi and Z. Hong-Ling, J. Xi, an Jiaotong Univ. (Med. Sci.), 313, 385 (2010).

6. Y.-L. Tan and C.-G. Liu, J. Mater. Sci. Mater. Med., 22, 1213 (2011).

7. Y.J. Lu, Adv. Drug Deliv. Rev., 54, 675 (2002).

8. S. Cai, S. Thati, T.R. Bagby, H.-M. Diab, N.M. Davies, M.S. Cohen and M.L. Forrest, J. Control. Rel., 146, 212 (2010).

9. H. Lee, C.H. Ahn and T.G. Park, Macromol. Biosci., 9, 336 (2009).

10. X.-B. Xiong, Z.S. Ma, R. Lai and A. Lavasanifar, Biomaterials, 31, 757 (2010).

11. M.E. Zambaux, F. Bonneaux, R. Gref, P. Maincent, E. Dellacherie, M.J. Alonso, P. Labrude and C. Vigneron, J. Control. Rel., 50, 31 (1998).

12. Yang Yan, Zhao Ying, Zhang Feng-Lan et al., Cancer Res. Prevention Treat., 38, 740 (2011). 